\title{
Der pulmonale Rundherd bei maligner Grunderkrankung - nicht zwingend eine Metastase des Primärtumors
}

\author{
Pulmonary Nodules in Underlying Malignant Diseases - \\ Not Necessarily Metastases of the Primary Tumour
}

Autoren

Institute
F. Beckers ${ }^{1}$, C. Ludwig ${ }^{1}$, J. Cerinza ${ }^{2}$, E. Stoelben ${ }^{1}$

Lungenklinik, Kliniken der Stadt Köln gGmbH, Köln

Universitätsklinik Freiburg, Abteilung Thoraxchirurgie, Freiburg eingereicht 19.5.2009 akzeptiert nach Revision 17. 8. 2009

\section{Bibliografie}

Dol http://dx.doi.org/ 10.1055/s-0029-1215109

Online-Publikation: 16.11. 2009

Pneumologie 2009; 63:

693-696 @ Georg Thieme

Verlag KG Stuttgart · New York ISSN 0934-8387

\section{Korrespondenzadresse} Dr. med. Frank Beckers Abteilung Thoraxchirurgie Lungenklinik Köln-Merheim Kliniken der Stadt Köln gGmbH Ostmerheimer Str. 200 51109 Köln beckersf@kliniken-koeln.de

\section{Zusammenfassung \\ $\nabla$}

Ziel: Die histologische Untersuchung von Lungenrundherden bei Patienten mit einem Malignom in der Anamnese. Darüber hinaus ein Vergleich der Anzahl der präoperativ im CT bestimmten Rundherde mit der Zahl der intraoperativ nachgewiesenen Befunde.

Patienten und Methoden: Zwischen 1998 und 2003 wurde bei 276 Patienten mit einer Tumoranamnese eine Resektion pulmonaler Rundherde durchgeführt. Anhand der Patientenakten wurde die Histologie des Primärtumors, die Histologie des Rundherdes sowie die Anzahl der präoperativen Rundherde und Zahl der intraoperativ verifizierten und resezierten Rundherde aufgezeichnet.

Ergebnisse: Von den 276 resezierten Patienten waren 161 Männer (58,1\%). Das Alter lag im Median bei 62 Jahren (21 - 86 Jahre). In jeweils 110 Fällen erfolgte die linksseitige bzw. rechtsseitige Thorakotomie (je 39,8\%), in 56 Fällen erfolgte ein beidseitiger Eingriff (20,4\%). In 15,2\% d. F. war die Histologie der Rundherde benigne, in 8,6\% d.F. zeigte sich ein primäres Bronchialkarzinom im Stadium I, in 74,4\% d.F. bestätigte sich die Metastase des aus der Vorgeschichte bekannten primären Karzinoms.

Schlussfolgerung: Isolierte Lungenrundherde bei Patienten mit Malignomanamnese sollten immer zur weiteren histologischen Abklärung reseziert werden. Bei multiplen Lungenrundherden sollte im Zweifelsfall die histologische Sicherung durch Resektion angestrebt werden.

\section{Einleitung}

$\nabla$

Bei Patienten mit einem malignen Tumor in der Vorgeschichte kommt es im Verlauf nicht selten zu Lungenmetastasen. Sollten im Rahmen der Primärbehandlung oder der Tumornachsorge bei diesen Patienten ein oder mehrere Lungenrund-

\section{Abstract \\ $\nabla$}

Background: In this study the histological examination of pulmonary nodules and a comparison of the number of pre-, intra- and post-operative lung metastases in patients with a previous history of malignant disease were assessed.

Patients and Methods: Between 1998 and 2003 we operated on 276 patients with suspected lung metastases. The histology of the primary tumour, the number of preoperatively diagnosed nodules, the number of lesions removed during surgery and the number of histologically confirmed metastases of 276 patients are presented.

Results: In 276 patients a resection was performed. 161 of the patients were men (58.1\%). The median age was 62 years (range: $21-86$ years). In 110 cases a left-sided thoracotomy was performed (39.8\%), in another 110 cases a right-sided thoracotomy was performed and in 56 cases we perfomed a bilateral thoracotomy (20.4\%). In $15.2 \%$ the histology of the resected nodules was benign. In $8.6 \%$ of the cases the histological examination showed a primary lung cancer in stage I, in $74.4 \%$ of the cases the histology confirmed a metastasis of the primary cancer.

Conclusions: Solitary pulmonary nodules in patients with a previous history of malignant disease should always be resected for histological examination. Multiple pulmonary nodules should be histologically reappraised if there is any doubt about the entity.

herde diagnostiziert werden, ist die Resektion des pulmonalen Befundes grundsätzlich indiziert. Voraussetzung für die Resektion ist der Ausschluss eines Lokalrezidivs des Primärtumors, keine weiteren Metastasen in anderen Organen und die Möglichkeit zur vollständigen Resektion des pulmonalen Befundes. Die Thorakotomie mit 


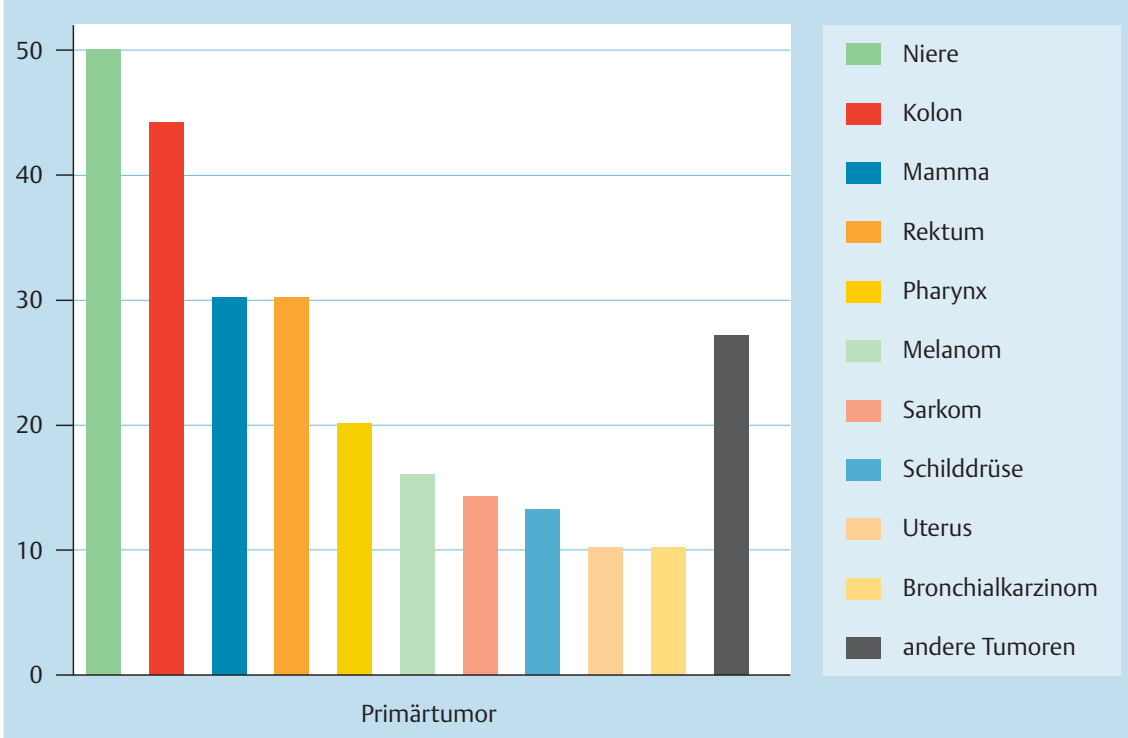

Abb. 1 Histologie des Primärtumors.

Palpation der Lunge ist hierbei die Operationsmethode der Wahl zur Resektion solitärer oder multipler Lungenmetastasen. Grundsätzlich wird die Palpation der Lunge gefordert, um im CT nicht detektierte Herde zu erfassen [1].

Die Publikationen über resezierte Lungenmetastasen legen ihren Fokus in der Analyse nahezu ausschließlich auf die tatsächlich histologisch gesicherten Metastasen. Es liegen nur wenige valide Ergebnisse über den Anteil an benignen Rundherden bei Patienten mit einer Karzinomanamnese vor.

Das Ziel dieser retrospektiven Studie ist es, die Heterogenität der histologischen Befunde bei pulmonalen Rundherden nach maligner Vorerkrankung zu untersuchen. Es wird der Anteil tatsächlicher Metastasen, bezogen auf die Gesamtzahl der untersuchten pulmonalen Rundherde, bestimmt. Darüber hinaus wurde die Zahl der präoperativ mittels CT-Thorax detektierten Metastasen mit der operativ explorierten Zahl verglichen.

Ein weiteres Ziel dieser retrospektiven Studie ist es, die Rolle der intraoperativen Palpation bei Patienten mit im Spiral-CT nachgewiesenen Rundherden, bei bekannter maligner Grunderkrankung, zu untersuchen.

Nach Einführung der Video-assistierten Thorakoskopie (VATS) erscheint uns diese Methode grundsätzlich ein geeignetes Verfahren zur Lungenmetastasenresektion zu sein [2-12]. Die periund postoperative Belastung für den Patienten ist bei diesem Verfahren in Vergleich zur Thorakotomie geringer, jedoch ist die intraoperative manuelle Palpation der Lunge über den minimalinvasiven Zugang nicht möglich.

\section{Patienten und Methoden \\ $\nabla$}

Zwischen 1998 und 2003 führten wir bei 281 Patienten mit Tumoranamnese eine Resektion pulmonaler Rundherde durch. Die Daten wurden anhand der Patientenakte retrospektiv erhoben. Die Daten waren in 276 Fällen vollständig aus der Akte zu entnehmen. Die Histologie des Primärtumors ( $\mathbf{A}$ bb. 1), die Zahl der präoperativ diagnostizierten pulmonalen Rundherde, die Anzahl der operativ entfernten Rundherde und der Anteil histologisch bestätigter Metastasen wurden aufgezeichnet ( $\bullet$ Abb. 2). Das präoperative Staging umfasste bei allen Patienten die Röntgenthoraxaufnahme, eine CT des Thorax, die Lungenfunktion so- wie eine arterielle Blutgasanalyse. Eine präoperative Bronchoskopie wurde bei allen Patienten durchgeführt.

Sämtliche Operationen wurden in Allgemeinnarkose unter EinLungenventilation durchgeführt. Der Zugangsweg war in Abhängigkeit von der Anzahl, der Lage und der Größe des pulmonalen Befundes entweder eine anterolaterale, eine posterolaterale oder eine bilaterale anterolaterale Thorakotomie. Nach manueller Palpation wurden die zu tastenden Rundherde nach Möglichkeit über eine gewebeschonende atypische Resektion entfernt. Je nach Befund wurden auch erweiterte Resektionen durchgeführt ( Tab. 1)

Zum Ende der Operation wurden je nach Resektionsausmaß eine oder zwei Thoraxdrainagen unter Sichtkontrolle platziert.

\section{Ergebnisse}

$\nabla$

Von den 276 resezierten Patienten waren 161 Männer (58,1\%) und 115 Frauen (41,9\%). Das Alter lag bei 21 - 86 Jahren (median 62 Jahre). Die Entität des Primärtumors variierte, wie in $\bullet$ Abb. 1 dargestellt. Die Thorakotomie erfolgte in 110 Fällen rechts und in 110 Fällen links (je 39,8\%). In 56 Fällen erfolgte ein beidseitiger Eingriff (20,2\%). Die Art der durchgeführten Resektion ist in

- Tab. 1 dargestellt.

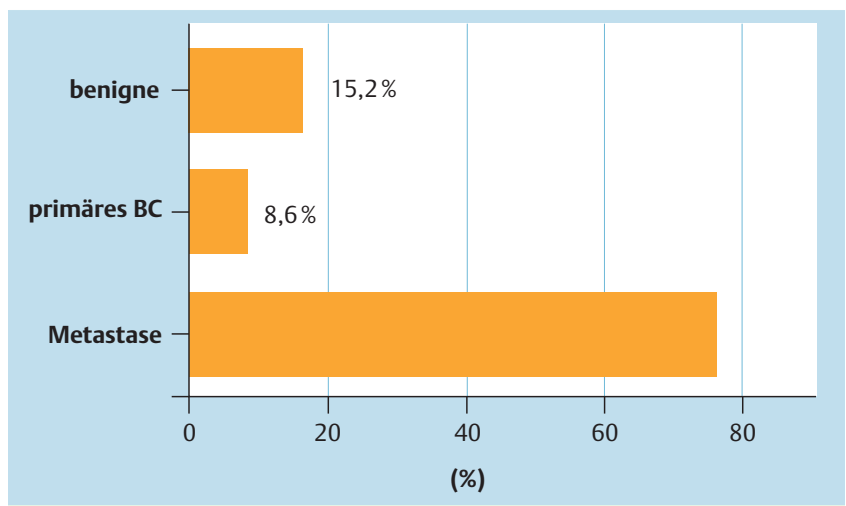

Abb. 2 Histologie der Resektate. 


\begin{tabular}{|l|l|} 
& $\begin{array}{l}\text { Prozentualer } \\
\text { Anteil (\%) }\end{array}$ \\
$\begin{array}{l}\text { Operationsseite } \\
\text { rechts }\end{array}$ \\
links & 39,8 \\
beidseits & 39,8 \\
\hline Resektionsmethode & 20,2 \\
atypische Resektion & 53,5 \\
Segmentresektion & 26,8 \\
Lobektomie & 12,3 \\
Pneumonektomie & 2,5 \\
Segment + atypisch & 2,8 \\
Lobektomie + atypisch & 1,4 \\
\hline
\end{tabular}

Tab. 1

OP-Seite und

Resektions-

methode.
In drei Fällen $(1,8 \%)$ wurden anstelle von intrapulmonalen Metastasen Pleura- oder Lymphknotenmetasten gefunden. In 42 Fällen $(15,2 \%)$ des Patientenkollektives mit maligner Vorgeschichte erwiesen sich die präoperativ gefundenen, einzelnen, aber auch multiplen Lungenrundherde als benigne, sodass sich bei diesen Patienten ein metastasiertes Tumorleiden nicht bestätigte.

Zeigte sich in der präoperativen CT-Thoraxuntersuchung ein einzelner pulmonaler Rundherd, so konnte intraoperativ in 11 von insgesamt 159 Fällen (6,9\%) mehr als eine Metastase intrapulmonal gesichert werden, in 26 von 159 Fällen (16,4\%) mit präoperativ nachgewiesenem solitärem Lungenrundherd konnte in der histologischen Aufarbeitung eine Metastase ausgeschlossen werden.

In den 117 Fällen mit präoperativ nachgewiesenen multiplen Lungenrundherden konnte in 10 Fällen (8,6\%) eine Malignität sämtlicher Rundherde histologisch ausgeschlossen werden.

In 10 Fällen (8,6\%) des Gesamtkollektives erbrachte die histologische Untersuchung der resezierten Rundherde den Nachweis eines primären Bronchialkarzinoms.

Bei den 117 Patienten mit präoperativem Nachweis von multiplen Lungenrundherden zeigten sich in 44 Fällen $(37,6 \%)$ intraoperativ weniger Metastasen als präoperativ bildmorphologisch nachgewiesen wurden. In 32 Fällen (27,4\%) fanden sich intraoperativ mehr Metastasen als CT-morphologisch erwartet ( Tab. 2).

\section{Diskussion}

Die Resektion von Lungenmetastasen ist in der Behandlung von malignen Tumoren etabliert. Bei Patienten mit einem krankheitsfreien Intervall von mehr als 36 Monaten und singulärer Metastase ist eine Prognoseverbesserung durch die Metastasenresektion nachgewiesen [13-15].

In der retrospektiven Auswertung von 276 Fällen mit pulmonalen Rundherden und maligner Vorerkrankung zeigten sich in 15,2\% der Fälle die Rundherde in der histopathologischen Unter- suchung benigne. In 8,6\% der Fälle konnte die histopathologische Aufarbeitung der Resektate ein primäres Bronchialkarzinom nachweisen. In der Addition konnte in $23,8 \%$ der Fälle ein metastasiertes Tumorleiden ausgeschlossen werden. Auch bei den Patienten mit multiplen pulmonalen Rundherden im CT zeigten sich in 10/117 Fällen (8,5\%) keine pulmonalen Metastasen. Diese Zahlen korrespondieren mit den Daten einer aktuellen Arbeit aus Japan [16].

Aufgrund dieser Daten ist bei Patienten mit maligner Vorerkrankung im geringsten Zweifel, besonders bei solitärem Lungenrundherd, aber auch bei isoliertem Lungenbefund, ohne weitere Organmanifestationen die Resektion zur histologischen Abklärung indiziert. Keinesfalls sollte ohne Histologiesicherung eine systemische Therapie begonnen werden.

Die Rolle des PET-CTs in der Diagnostik der solitären oder multiplen Lungenrundherde ist gut untersucht [17-20]. Eine aktuelle Metaanalyse zur Untersuchung von Lungenrundherden konnte erneut keinen signifikanten Unterschied bezüglich der Sensitivität und Spezifität für das PET im Vergleich zur dynamischen CT zeigen [21].

Darüber hinaus verglichen wir anhand der Daten die Anzahl der präoperativ in der CT-Untersuchung erfassten Zahl der pulmonalen Rundherde mit der Zahl der intraoperativ tatsächlich gefundenen pulmonalen Raumforderungen. Bei den Patienten mit einer solitären pulmonalen Raumforderung im CT zeigte sich in nur 6,9\% der Fälle bei der operativen Exploration mehr als eine Metastase. Die VATS kann somit grundsätzlich als Resektionsmethode der Wahl bei präoperativer Detektion eines solitären Lungenrundherdes angesehen werden. Die Grenzen der Technik stellen die Tumorgröße $(<3 \mathrm{~cm})$ und die Lage des Herdes dar. Um eine VATS-Resektion sicher durchführen zu können, sollte der Tumor peripher im Lungenparenchym gelegen sein, da es bei zentraler Lage schwierig sein kein, den isolierten Herd ohne manuelle Palpation zu lokalisieren. Die VATS ist eine sichere OP-Technik mit geringer Belastung für den Patienten. Darüber hinaus erlaubt sie die wiederholte Resektion bei erneuter Metastasierung und bringt eine definitive histologische Klärung des Befundes, sodass nicht indizierte Therapien vermieden werden können [22-26]. Bei Vorliegen von mehr als einer Raumforderung im prä-operativen CT zeigten sich in 27,4\% mehr als die im CT detektierten Metastasen und in 37,6\% weniger Metastasen als präoperativ angenommen. Da bei mehr als einem Viertel der Patienten durch das manuelle Austasten der Lunge weitere, nicht erwartete Herde gefunden wurden, ist bei multiplen pulmonalen Rundherden die Thorakotomie als Resektionsmethode der Wahl zu betrachten. Die CT-morphologisch detektierten Herde können nicht mit ausreichender Sicherheit ohne manuelles Austasten der gesamten Lunge minimalinvasiv reseziert werden.

$\begin{array}{llll} & \text { Patienten } & \begin{array}{l}\text { intraoperativ } \\ \text { Rundherde }\end{array} & \begin{array}{l}\text { histologisch } \\ \text { Metastase }\end{array} \\ & & 0=3 & 0=26(16 \%) \\ \text { präoperativim CT } & \mathrm{n}=159 & 1=130 & 1=122 \\ \text { 1 Metastase } & & >1=26(16 \%) & >1=11(7 \%) \\ & & {[<\mathrm{CT}]=12} & {[<\mathrm{CT}]=44} \\ \text { präoperativim CT } & \mathrm{n}=117 & {[=\mathrm{CT}]=45} & {[\mathrm{CT}]=41} \\ >\text { 1 Metastase } & & [>\mathrm{CT}]=60] & {[>\mathrm{CT}]=32(27 \%)}\end{array}$

Tab. 2 Anzahl der CT-morphologisch detektierten Rundherde verglichen mit den intraoperativen Befunden und der Anzahl der histologisch bestätigten Metastasen. 


\section{Fazit für die Praxis}

$\nabla$

Da 15,2\% aller pulmonalen Rundherde gutartig waren und zusätzlich 8,6\% der Befunde einem primären Bronchialkarzinom entsprachen, empfehlen wir dringend die Resektion zur histologischen Abklärung neu aufgetretener pulmonaler Rundherde bei Karzinomanamnese. Ein solitärer Lungenrundherd ist bis zum Beweis des Gegenteils nicht als Metastase anzusehen, gehört jedoch zwingend weiter abgeklärt. Da auch bei Patienten mit multiplen Rundherden in 8,5\% keine Metastase nachgewiesen werden konnte, sollte auch in diesen Fällen eine Abklärung erfolgen. Bei solitärer Lungenmetastase zeigte sich intraoperativ lediglich in 7\% der Fälle mehr als der präoperativ detektierte Herd. Daher ist die VATS-Resektion die Operationsmethode der Wahl bei solitärem Befund im CT-Thorax. Bei einem Viertel der Patienten mit multiplen Metastasen im präoperativen CT zeigten sich intraoperativ mehr Herde als erwartet, sodass hier die Thorakotomie mit manueller Palpation der Lunge zur vollständigen Resektion notwendig ist.

\section{Interessenkonflikte}

$\nabla$

Es besteht kein Interessenkonflikt.

\section{Literatur}

1 McCormack PM, Bains MS, Begg CB et al. Role of video-assisted thoracic surgery in the treatment of pulmonary metastases: results of a prospective trial. Ann Thorac Surg 1996; 62: 213 - 216; discussion 216 217

2 Mitruka S, Landreneau RJ, Mack MJ et al. Diagnosing the indeterminate pulmonary nodule: percutaneous biopsy versus thoracoscopy. Surgery 1995; 118: 676-684

3 Cardillo G, Regal M, Sera F et al. Videothoracoscopic management of the solitary pulmonary nodule: a single-institution study on 429 cases. Ann Thorac Surg 2003; 75: 1607 - 1611

4 Downey RJ, McCormack P, LoCicero J 3rd. The Video-Assisted Thoracic Surgery Study Group. Dissemination of malignant tumors after videoassisted thoracic surgery: a report of twenty-one cases. J Thorac Cardiovasc Surg 1996; 111: $954-960$

5 Mutsaerts EL, Zoetmulder FA, Meijer S et al. Long term survival of thoracoscopic metastasectomy vs metastasectomy by thoracotomy in patients with a solitary pulmonary lesion. Eur J Surg Oncol 2002; 28: $864-868$

6 Schwarz RE, Posner MC, Plunkett MB et al. Needle-localized thoracoscopic resection of indeterminate pulmonary nodules: impact on management of patients with malignant disease. Ann Surg Oncol 1995; 2: $49-55$

7 Erasmus JJ, Connolly JE, McAdams HP, Roggli VL. Solitary pulmonary nodules: Part I. Morphologic evaluation for differentiation of benign and malignant lesions. Radiographics 2000; 20: $43-58$
8 Ginsberg RJ. The solitary pulmonary nodule: can we afford to watch and wait? J Thorac Cardiovasc Surg 2003; 125: 25-26

9 Khouri NF, Meziane MA, Zerhouni EA, Fishman EK, Siegelman SS. The solitary pulmonary nodule. Assessment, diagnosis, and management. Chest 1987; 91: 128-133

10 Ost D, Fein A. Evaluation and management of the solitary pulmonary nodule. Am J Respir Crit Care Med 2000; 162: 782 - 787

11 Tan BB, Flaherty KR, Kazerooni EA, Iannettoni MD. The solitary pulmonary nodule. Chest 2003; 123: 89S-96S

12 Ludwig C, Zeitoun M, Stoelben E. Video-assisted thoracoscopic resection of pulmonary lesions. Eur J Surg Oncol 2004; 30: 1118 - 1122

13 Friedel G, Pastorino $U$, Buyse $M$ et al. Resection of lung metastases: long-term results and prognostic analysis based on 5206 cases - the International Registry of Lung Metastases. Zentralbl Chir 1999; 124: 96- 103

14 Friedel G, Pastorino U, Ginsberg RJ et al. Results of lung metastasectomy from breast cancer: prognostic criteria on the basis of 467 cases of the International Registry of Lung Metastases. Eur J Cardiothorac Surg 2002; 22: $335-344$

15 Thomford NR, Woolner LB, Clagett OT. The Surgical Treatment of Metastatic Tumors in the Lungs. J Thorac Cardiovasc Surg 1965; 49: 357-363

16 Nakajima J, Murakawa T, Fukami T et al. Is finger palpation at operation indispensable for pulmonary metastasectomy in colorectal cancer? Ann Thorac Surg 2007; 84: 1680-1684

17 Aquino SL, Kuester LB, Muse VV et al. Accuracy of transmission CT and FDG-PET in the detection of small pulmonary nodules with integrated PET/CT. Eur J Nucl Med Mol Imaging 2006; 33: 692 - 696

18 Reinhardt MJ, Wiethoelter N, Matthies A et al. PET recognition of pulmonary metastases on PET/CT imaging: impact of attenuation-corrected and non-attenuation-corrected PET images. Eur J Nucl Med Mol Imaging 2006; 33: 134-139

19 De Wever W, Meylaerts L, De Ceuninck L et al. Additional value of integrated PET-CT in the detection and characterization of lung metastases: correlation with CT alone and PET alone. Eur Radiol 2007; 17: $467-473$

20 Yap CS, Czernin J, Fishbein MC et al. Evaluation of thoracic tumors with 18 F-fluorothymidine and 18 F-fluorodeoxyglucose-positron emission tomography. Chest 2006; 129: 393-401

21 Cronin P, Dwamena BA, Kelly AM, Carlos RC. Solitary pulmonary nodules: meta-analytic comparison of cross-sectional imaging modalities for diagnosis of malignancy. Radiology 2008; 246: 772 - 782

22 Welter $S$, Jacobs J, Krbek $T$ et al. Long-term survival after repeated resection of pulmonary metastases from colorectal cancer. Ann Thorac Surg 2007; 84: $203-210$

23 Jaklitsch MT, Mery CM, Lukanich JM et al. Sequential thoracic metastasectomy prolongs survival by re-establishing local control within the chest. J Thorac Cardiovasc Surg 2001; 121: 657-667

24 Kandioler D, Kromer E, Tuchler $\mathrm{H}$ et al. Long-term results after repeated surgical removal of pulmonary metastases. Ann Thorac Surg 1998; 65: 909-912

25 De Giacomo T, Rendina EA, Venuta F et al. Thoracoscopic resection of solitary lung metastases from colorectal cancer is a viable therapeutic option. Chest 1999; 115: $1441-1443$

26 Stammberger U, Steinacher C, Hillinger S et al. Early and long-term complaints following video-assisted thoracoscopic surgery: evaluation in 173 patients. Eur J Cardiothorac Surg 2000; 18: 7-11 